Proc. Indian Acad. Sci. (Earth Planet. Sci.), Vol. 95, No. 1, March 1986, pp. 75-82

(C) Printed in India.

\title{
Quasi-specular sea scatter with modified reflection coefficient
}

\author{
RAJ KUMAR and ABHIJIT SARKAR \\ Meteorology and Oceanography Division, Space Applications Centre (ISRO), Ahmedabad \\ 380053, India
}

MS received 8 July 1985 ; revised 16 December 1985

\begin{abstract}
The paper deals with the relationship between the sea surface wind speed $(U)$ and the backscattering coefficient $\left(\sigma^{\circ}\right)$ for microwave frequencies and quasi specular range of angles. Valenzuela's effective reflection coefficient based on the theory of scattering of electromagnetic waves from rough dielectric surfaces has been introduced in the expression of backscattering coefficient and the final result compared with the $\sigma^{\circ}-U$ relationship derived from SEASAT scatterometer and concurrent sea truth data.
\end{abstract}

Keywords. Scattering; SEASAT; sea surface wind speed; quasi specular reflection.

\section{Introduction}

Satellite scatterometry has made significant advancement in the last two decades. Scatterometers onboard the satellites SKYLAB and SEASAT have mapped the oceanic wind on a global scale. Determination of the oceanic wind vector by scatterometers is possible because the backscattering coefficient at microwave frequencies is correlated to the ocean surface wind vector. This is explained by the mechanism of Bragg scattering for large incidence angles and quasi-specular reflection near normal incidence (Moore and Fung 1979). Normal incidence scattering coefficient measurements by altimeters have also been used to retrieve surface wind speed (Brown et al 1981).

Current scattering theories (Fung 1981) have explained the trends in the ocean backscatter measurements to a large extent. But considerable refinements and modifications are required before they can be used for definite remote sensing applications with confidence. In the present paper is given a modified expression of backscattering coefficient from rough ocean surface as a function of surface wind speed for quasi-specular range of angles. The modifications are made by using Valenzuela's expression $(1970,1978)$ for effective reflection coefficient for rough surfaces in the expression for backscattering coefficient in quasi-specular scattering (Barrick 1974). The angular dependence of the coefficients of the wind-backscatter relationship are generated as analytical functions.

\section{Quasi-specular sea scatter}

Based on the specular point model, which involves derivation of the average number of specular points for a two-dimensionally rough surface and the Gaussian curvature of these points, Barrick (1974) has developed a simple expression for the backscattering 
coefficient in the quasi-specular range of incidence angles (i.e., incidence angles upto $\left.20^{\circ}\right)$ :

$$
\sigma^{\circ}=\pi \sec ^{4} \theta P(-\tan \theta, 0)|R(0)|^{2},
$$

where,

$\theta=$ angle of incidence from the vertical, $P\left(\frac{\partial Z}{\partial x}, \frac{\partial Z}{\partial y}\right)=$ joint probability density function of the surface slopes,

$Z=$ surface height above the mean $(x y)$ plane, and

$R(0)=$ Fresnel reflection coefficient of the surface at normal incidence.

It has been assumed that the $x z$ plane is the incident plane.

It is reasonable to interpret (1) as the scattered signal, being merely proportional to the probability of the surface having regions tilted at slope $\tan \theta$ which make them normal to the incident ray. For an isotropic rough surface of Gaussian statistics, (1) becomes

$$
\sigma^{\circ}=\left(1 / S^{2}\right)|R(0)|^{2} \sec ^{4} \theta \exp \left(-\tan ^{2} \theta / S^{2}\right)
$$

where $S^{2}$ is the total variance of slopes.

\section{Effective reflection coefficient}

Barrick (1974) used the Fresnel reflection coefficient at normal incidence in the expression for $\sigma^{\circ}$ (2). However, the reflection properties of the surface can be significantly modified by the amplitude of the roughness present. Hence, for the reflection coefficient of a wind driven rough surface, an effective reflection coefficient should be used. Using Rice's theory for forward scattering of electromagnetic waves from a dielectric rough surface (Rice 1951), Valenzuela $(1970,1978)$ has derived the following expression for effective reflection coefficients:

where

$$
\begin{aligned}
& R_{\perp} \text { effective }=R_{\perp}+\frac{1}{2} \frac{\beta^{2} \gamma(\varepsilon-1)}{(\gamma+\eta)^{2}} I_{\perp} \\
& R_{\|} \text {effective }=R_{\|}-\frac{1}{2} \frac{\beta^{2} \gamma(\varepsilon-1) \varepsilon}{(\varepsilon \gamma+\eta)^{2}} I_{\|}
\end{aligned}
$$

$$
\begin{aligned}
R_{\perp}= & \frac{\gamma-\eta}{\gamma+\eta} ; \quad R_{\|}=\frac{\varepsilon \gamma-\eta}{\varepsilon \gamma+\eta} ; \\
I_{\perp}= & \int_{-\infty}^{\infty} \mathrm{d} p \int_{-\infty}^{\infty} \mathrm{d} q\left[\eta+\frac{\beta(\varepsilon-1)}{\mathrm{d}(p, q)}\left(q^{2} / D_{p q}-1\right)\right] W(p-\alpha \beta, q) ; \\
I_{\|}= & \int_{-\infty}^{\infty} \mathrm{d} p \int_{-\infty}^{\infty} \mathrm{d} q\left\{-\frac{\alpha}{\beta}[p \eta-\alpha c(p, q)]+\eta-\frac{\beta \alpha^{2} \varepsilon b(p, q)}{D_{p q}}\right. \\
& \left.+\frac{p \beta \alpha(\varepsilon-1) \eta b(p, q)}{\mathrm{d}(p, q) D_{p q}}-\frac{p \beta \alpha \eta}{D_{p q}}-\frac{\beta(\varepsilon-1) \eta^{2}}{\varepsilon \mathrm{d}(p, q)}\left(1-\frac{p^{2}}{D_{p q}}\right)\right\} W(p-\alpha \beta, q) ;
\end{aligned}
$$




$$
\begin{gathered}
\eta=\left(\varepsilon-\alpha^{2}\right)^{\frac{1}{2}} ; \quad \gamma=\cos \theta ; \alpha=\sin A ; \\
b(p, q)=\left(\beta^{2}-p^{2}-q^{2}\right)^{\frac{1}{2}} ; \quad c(p, q)=\left(\beta^{2} \varepsilon-p^{2}-q^{2}\right)^{\frac{1}{1}} ; \\
\mathrm{d}(p, q)=b(p, q)+c(p, q) ; \quad D_{p q}=b(p, q) c(p, q)+p^{2}+q^{2}
\end{gathered}
$$

Hence, for computations of the backscattering coefficient, the following expression has been used here:

$$
\sigma^{\circ}(\theta)=\frac{\left|R_{\text {effective }}(0)\right|^{2}}{S^{2}} \sec ^{4} \theta \exp \left(-\frac{\tan ^{2} \theta}{S^{2}}\right)
$$

It is realized that the choice of the sea spectrum (which is undergoing continuous refinement in recent times) will influence the final results. In this paper, the following specific form of the spectrum $W(K)$ is selected:

$$
W(K)= \begin{cases}C K^{4} /\left(\kappa^{2}+K^{2}\right)^{4}, K \leqslant K_{c} \\ 0 \quad, \quad K>K_{c}\end{cases}
$$

where $K$ is the wavenumber. Equation (6) represents a polynomial approximation of the so-called Pierson Moskowitz spectrum for the steady state response of the ocean surface to a surface wind speed $U$ and has been used earlier by Brown (1978) for studying scattering by composite random surfaces. The constant $\kappa$ is given by $\left(335.2 U^{4}\right), K$ in $\left(\mathrm{cm}^{-1}\right)$ and $U$ in $\mathrm{m} / \mathrm{sec}$, the spectral constant $C$ was taken to be 0.0046 and cut off wavenumber $K_{c}$ to be $12 \mathrm{~cm}^{-1}$.

The spectrum was selected merely for the sake of an example and a realistic spectrum should be used for exact evaluation.

\section{Comparison with SEASAT scatterometer results}

To evaluate the performance of the original Barrick's expression (2), and the expression with the modified reflection coefficient (5), backscattering coefficient values were computed for the SEASAT-A scatterometer (SASS) system parameters (Boggs 1982). SEASAT provided an opportunity to collect a large amount of calibrated scattering coefficient data. Britt and Schroeder (1984) developed a functional data base by merging large numbers of SASS measurements (35073 horizontal and 70744 vertical polarization measurement samples) with high quality surface truth wind data. The data base is represented by a multivariate regression equation by relating wind speed, relative wind direction and incidence angle, to backscattering coefficient:

$$
\begin{aligned}
& \sigma^{\circ}(\mathrm{d} b)=\sum_{i=1}^{12} b_{i} x^{f_{i}} y_{i} z^{h_{i}} \\
& \text { where } x=\sin \theta, \quad y=\log (U), \quad z=\cos \phi
\end{aligned}
$$

$\theta$ is the incidence angle and $\phi$ the relative wind direction.

The regression coefficients are given in table 1.

In figures 1-3 are illustrated the variations in the values of scattering coefficient over the angular range of 0 to $16^{\circ}$ for the upwind case for wind speed values of 5,15 and $20 \mathrm{~m} / \mathrm{sec}$ respectively. It should be noted that the effect of polarization and wind direction are insignificant in the quasi-specular range of angles. In all the cases, it is seen that Barrick's original expression (2) is closer to the SEASAT regression model curve for 
Table 1. Regression coefficients

\begin{tabular}{rrrrrr}
\hline & & & \multicolumn{3}{c}{$b_{l}$} \\
\hline 1 & $f$ & $g$ & $h$ & \multicolumn{1}{c}{$H$-pol } & \multicolumn{1}{c}{$V$-pol } \\
\hline 1 & 2 & 0 & 0 & -295.0534 & -313.9546 \\
2 & 3 & 0 & 0 & 267.0684 & 300.6422 \\
3 & 4 & 0 & 0 & $000 \cdot 0000$ & $000 \cdot 0000$ \\
4 & 0 & 1 & 0 & -004.4289 & $-004 \cdot 4752$ \\
5 & 2 & 1 & 0 & 93.9786 & 102.2082 \\
6 & 3 & 1 & 0 & 000.0000 & 000.0000 \\
7 & 4 & 1 & 0 & -109.9966 & -126.5153 \\
8 & 2 & 1 & 1 & 000.0000 & 000.0000 \\
9 & 2 & 1 & 2 & 7.7536 & 13.0239 \\
10 & 2 & 1 & 3 & 2.8942 & 0.9506 \\
11 & 2 & 1 & 4 & -002.0757 & -005.3607 \\
12 & 0 & 0 & 0 & 15.6307 & 15.9729 \\
\hline
\end{tabular}

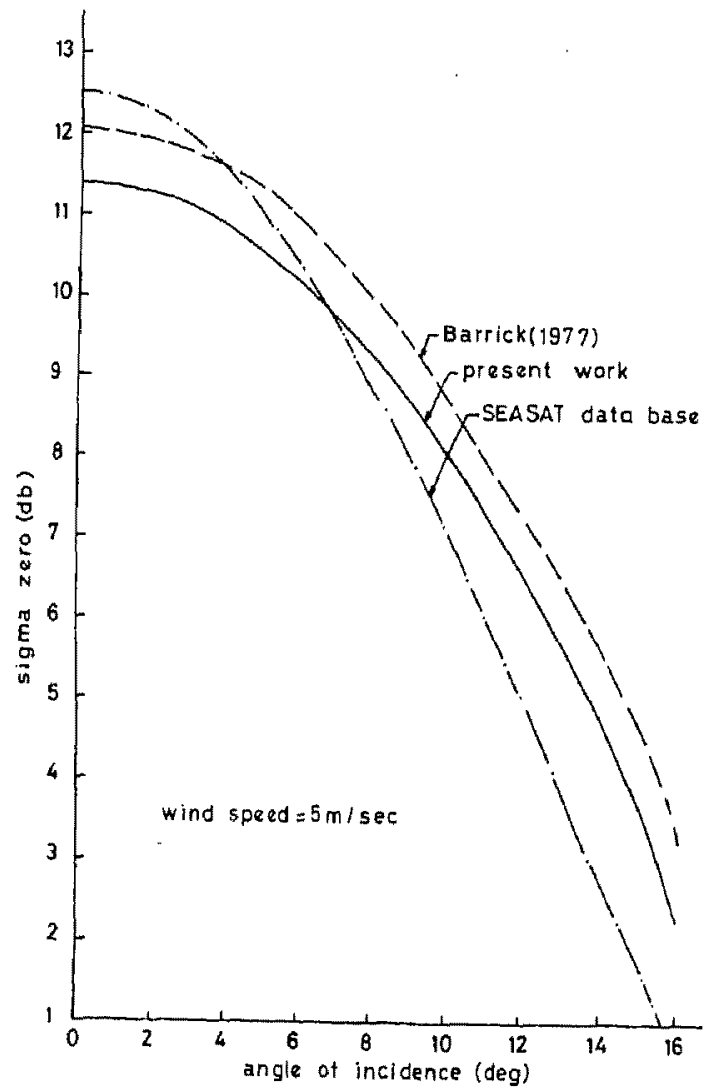

Figure 1. Angular variations of $\sigma^{\circ}$ for wind speed of $5 \mathrm{~m} / \mathrm{sec}$. 


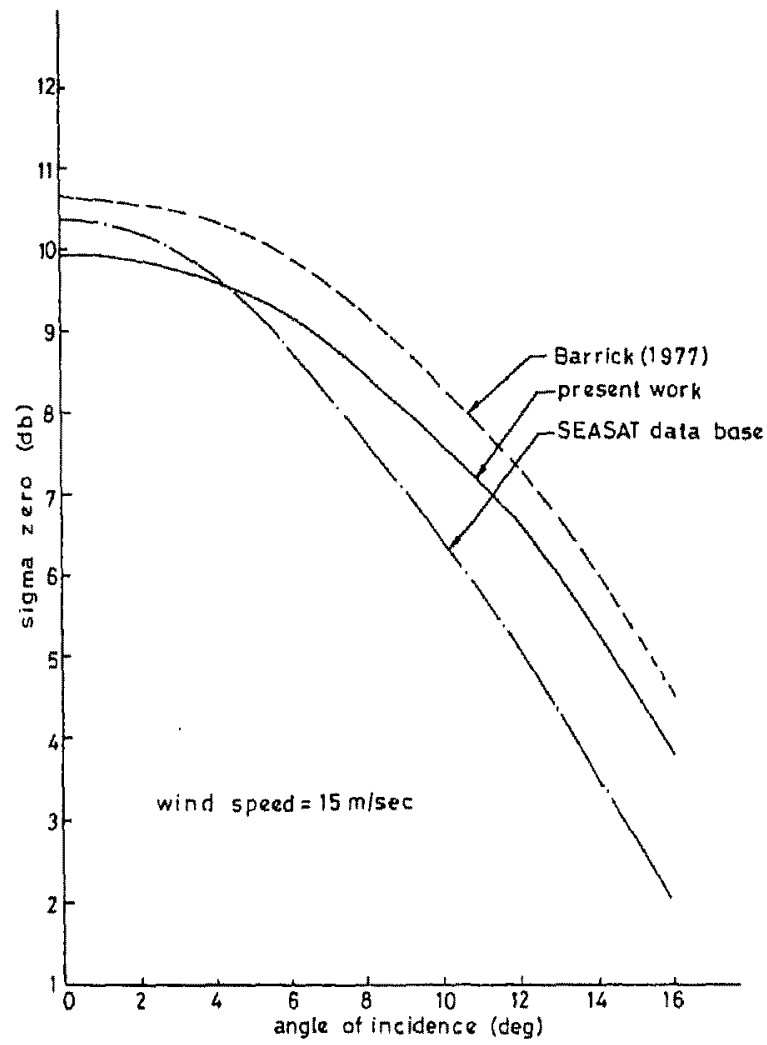

Figure 2. Angular variations of $\sigma^{\circ}$ for wind speed of $15 \mathrm{~m} / \mathrm{sec}$.

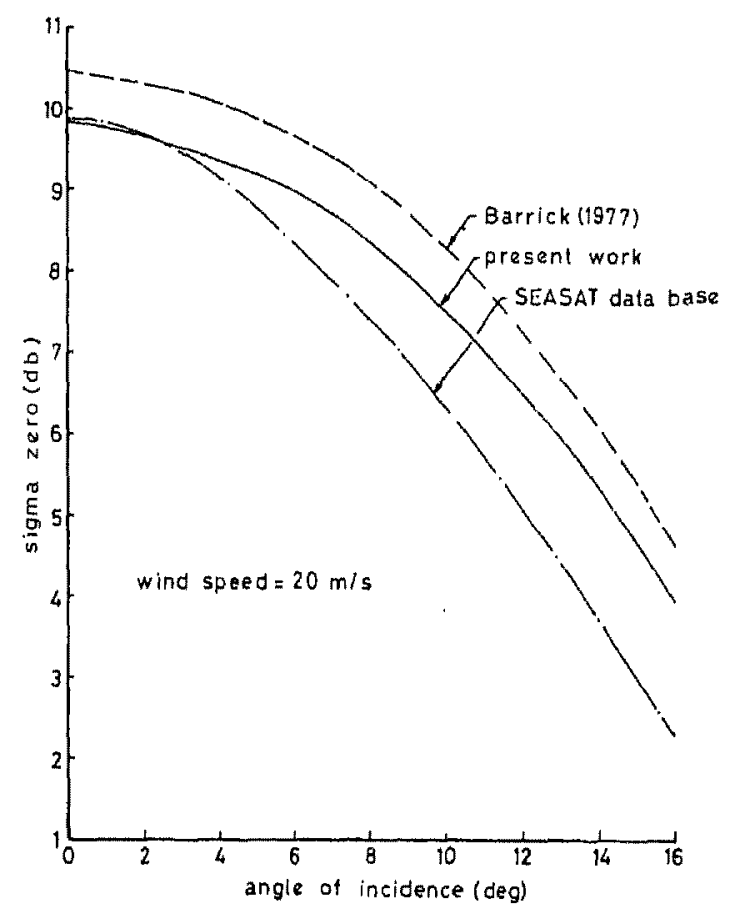

Figure 3. Angular variations of $\sigma^{\circ}$ for wind speed of $20 \mathrm{~m} / \mathrm{sec}$. 
angles upto about 7 degrees, while beyond that the modified expression provides more accurate results.

\section{Power law relationship}

A simple power law relationship of the type $\sigma^{\circ}=A(\theta) . U^{\left.\mathrm{B}()^{(}\right)}$is found to be a fairly good representation of the relationship between surface wind speed and the backscattering coefficient. The coefficients $A$ and $B$ would depend on the frequency, polarization and angle of observation. It is possible to generate analytical functions for $A$ and $B$ through the method of numerical curve fitting. For the case of quasi-specular sea scatter as given by Barrick (1974), the angle dependent functions $A(\theta)$ and $B(\theta)$ were generated for the $X$ band (Sarkar and Bhaduri 1984). Following similar procedures, functions $A(0)$ and $B(\theta)$ have been generated for the modified backscattering coefficient, given by (5). In figure 4 is shown the power law relationship between $\sigma^{\circ}$ and $U$ on a logarithmic scalc for different angles of incidence. In figure 5 is illustrated the angular dependence of $A$ and $B$ for sass frequency. The following analytical functions have been obtained for $A(U)$ and $B(\theta):$

$$
\begin{aligned}
& A(\theta)=\sum_{i=0}^{4}\left(A_{i} \theta^{i}\right), \\
& B(\theta)=\sum_{i=0}^{4}\left(B_{i} \theta^{i}\right)
\end{aligned}
$$

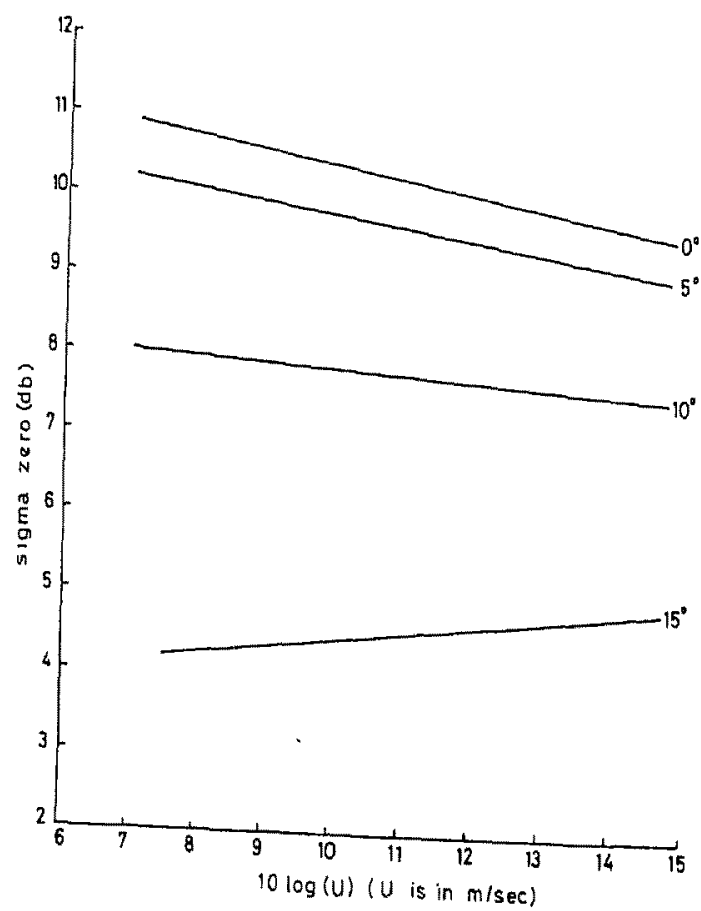

Figure 4. $\sigma^{\circ}$ as a function of wind spced for different angles of incidence. 


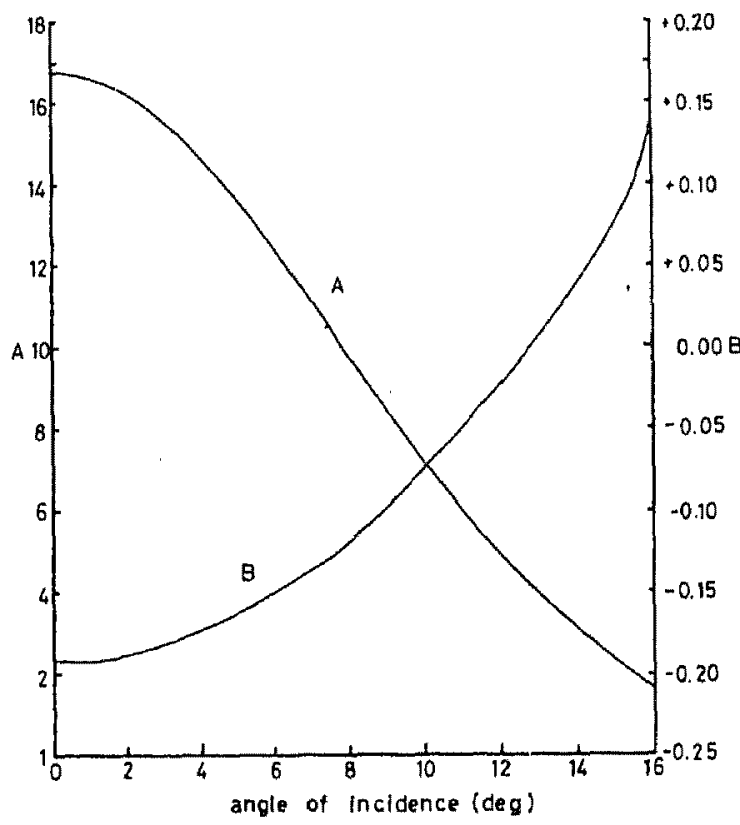

Figure 5. Angular variations of the coefficients $A$ and $B$.

Table 2. Coefficients $A_{i}$ and $B_{i}$.

\begin{tabular}{rrrrrr}
\hline$i$ & 0 & 1 & 2 & 3 & 4 \\
\hline$A$ & $16 \cdot 5795$ & 0.1964 & -0.2146 & 0.01178 & $-0.1704 \mathrm{E}-03$ \\
$B$ & -0.1932 & $-0.1019 \mathrm{E}-02$ & 3.77379 & -0.163244 & 2.97941 \\
\hline
\end{tabular}

where $\theta$ is in degrees in (8) and in radians in (9). The coefficients $A$ and $B$ are given in table 2. Fourth degree polynomials are found to give excellent fits with RMS errors of only $0.65 \%$ and $0.067 \%$ for $A(\theta)$ and $B(\theta)$ respectively.

\section{Results and discussion}

Barrick (1974) found a fairly good agreement of measured scattering coefficient values at normal incidence with those obtained through his quasi-specular model. However, at angles away from normal, considerable disagreement is noticed (Valenzuela 1978). This is also seen in figures 1-3. Possible remedies lie in using mean square slopes of large waves (i.e. for the case where gravity-capillary waves have been suppressed) and modified reflection coefficient (as it has been done in this paper) in the model for the quasi-specular sea scatter. Figures 1-3 indeed clearly illustrate the improvement brought about by the modified reflection coefficient over the angles away from normal. Modifications due to the spray and foam can also be introduced if an appropriate parametric form can be found for them. Studies (Huang et al 1984) are being pursued to 
evolve realistic functional forms of probability density functions of ocean slope and elevation, which have been elusive owing to the nonlinear nature of ocean waves. In figures 1-3 one finds an improvement of about $1 \mathrm{~dB}$ over the quasi-specular model. Effectively this will bring about improvement of the order of $15 \%$ in retrieved wind speed.

\section{Acknowledgements}

The authors acknowledge encouragement and guidance from Dr T A Hariharan. They wish to thank Dr P C Pandey and other colleagues for useful discussions.

\section{References}

Barrick D E 1977 IEEE Trans. Antennas Propag. AP-22 135

Boggs D H 1982 Seasat geophysical data record (GDR): Users handbook

Britt C L Jr and Schroeder L C 1984 Proceedings of International Geoscience and Remote Sensing Symposium IGARss'84 Strasbourg (ESA SP-215) 623

Brown G S 1978 IEEE Trans. Antennas Propag. AP-26 472

Brown G S, Stanley H R and Roy N A 1981 IEEE J. Oceanic Eng. OE-6 59

Fung A K 1981 Proceedings of ESA-EARSel workshop held at Alpabech, Austria (ESA SP-166) 71

Huang N E, Long S R and Bliven L F 1984 J. Geophys. Res. 891961

Moore R K and Fung A K 1979 Proc. IEEE 671504

Rice 1951 Comm. Pure Appl. Math. 4451

Sarkar A and Bhaduri L 1984 Proc. Indian Acad Sci. (Earth Planet. Sci.) 93111

Valenzuela G R 1970 Proc. IEEE 581279

Valenzucla G R 1978 Boundary-Layer Meterol, 1361 